\title{
Pleomorphic Xanthoastrocytoma with Oligodendroglioma-Like Areas with Negative 1p19q Co-Deletion
}

\author{
Deepa Goel ${ }^{1}$ Shobhna Sharma ${ }^{1}$ Aditya Gupta ${ }^{2}$ \\ ${ }^{1}$ Department of Pathology, Artemis Hospitals, Gurugram, Haryana, India \\ Address for correspondence Deepa Goel, MBBS, MD, Artemis \\ ${ }^{2}$ Department of Neurosurgery, Artemis Hospitals, Gurugram, \\ Haryana, India \\ Hospitals, Sector -34, Gurugram, Haryana, India \\ (e-mail: deepa.goel3@gmail.com).
}

Arq Bras Neurocir 2021;40(2):e186-e189.

\begin{abstract}
Keywords

- pleomorphic xanthoastrocytoma

- mixed glioma

- composite glioma

- oligodendroglioma

- 1p19q

The most common mixed glioma encountered in routine surgical practice is oligoastrocytoma (OA); however, its is currently considered a vanishing entity. The 2016 classification of the World Health Organization (WHO) discourages the diagnosis of tumors as mixed glioma. The recommendations are that diffuse gliomas, including those with mixed or ambiguous histological features, should be subjected to molecular testing. Dual-genotype OAs are not yet a distinct entity or variant in the classification. We report a case of mixed glioma: a pleomorphic xanthoastrocytoma (PXA) mixed with an oligodendroglioma. The immunohistochemistry (IHC) pattern of isocitrate dehydrogenase 1 (IDH1) negativity with retained nuclear expression of the alpha-thalassemia $x$-linked intellectual disability syndrome (ATRX) protein, and 1p19q co-deletion negativity in both the components enabled its identification as a mixed glioma rather than a collision tumor. To the best of our knowledge, the case herein presented is the fourth case of PXA with oligodendroglioma. Out of the other three reported cases, only one was of a collision tumor with a dual genotype, and the other two showed similar molecular signatures in both components. The present article discusses the histological, immunohistochemical and molecular features of the aforementioned case.
\end{abstract}

\section{Introduction}

Pleomorphic xanthoastrocytoma (PXA) is uncommon, and it represents less than $1 \%$ of all brain tumors. ${ }^{1}$ Tumors have heterogeneous histological appearance, including spindle cells, lipidized cells, giant cells, eosinophilic granular bodies, and perivascular lymphocytes. The variant forms in which PXA exhibits a mixed histologic pattern have been described, and they include ganglioglioma (GG), dysembryoplastic neuroepithelial tumor (DNET), oligodendroglioma, diffuse astrocytoma (DA), and epithelioid glioneuronal tumor (EGT). ${ }^{2-8}$ Perry et al. ${ }^{4}$ were the first to describe an oligodendroglioma with PXA, in which the oligodendroglioma component comprised $90 \%$ of the tumor, but $1 \mathrm{p} 19 \mathrm{q}$ co-deletion was negative. They concluded that PXA represented the astrocytic component of oligoastrocytoma (OA). After a decade, Hattab et al. ${ }^{5}$ described a collision tumor with genetically distinct areas of PXA and oligodendroglioma. Murakami et al. ${ }^{6}$ reported a case of oligodendroglioma with a PXA-like microlesion with similar molecular signature. The case herein presented is the fourth case of composite PXA and oligodendroglioma with negativity of 1p19q co-deletion in both components. received

August 13, 2020

accepted

DOI https://doi.org/

$10.1055 / \mathrm{s}-0040-1722245$.

ISSN $0103-5355$. 


\section{Case History}

A 31-year-old man presented with a 15-year history of seizures. The frequency of the seizures was of $\sim 6$ to 7 episodes a month, which were followed by loss of consciousness and right-hand automatism. He was on antiepileptic medications. A contrast-enhanced magnetic resonance inaging (MRI) scan of the brain revealed an intra-axial multi-loculated subcortical solid cystic mass lesion in the right temporal lobe with expansion of the temporal lobe and effacement of the adjacent sulci. The solid portion of the lesion was iso-hypointense on T1, slightly hyperintense on $\mathrm{T} 2$, and showed enhancement on post contrast. Internal blooming areas were observed within the lesion. The cystic component was hypointense on $\mathrm{T} 1$ and hyperintense on $\mathrm{T} 2$ and on fluid-attenuated inversion recovery (FLAIR images). A midline shift of $4 \mathrm{~mm}$ toward the left side was observed. The findings were suggestive of a low-grade glioma - LGG/PXA. The patient underwent right temporal craniotomy and decompression of the mass.

A biopsy of superficial and deep tissues was performed. Sections of the deep-tissue biopsy showed two different and intermingled morphologies. There were nodules of round monomorphic cells (oligodendroglial areas) mixed with other lesions, showing spindle to lipidized cells, with highly pleomorphic cells, including binucleated and multinucleated cells and perivascular lymphocytic infiltrate (PXA). No mitosis or necrosis were observed (-Fig. 1a). The superficial-tissue biopsy showed a mainly normal brain parenchyma with a tiny focus of tumor with round monomorphic cells with clear

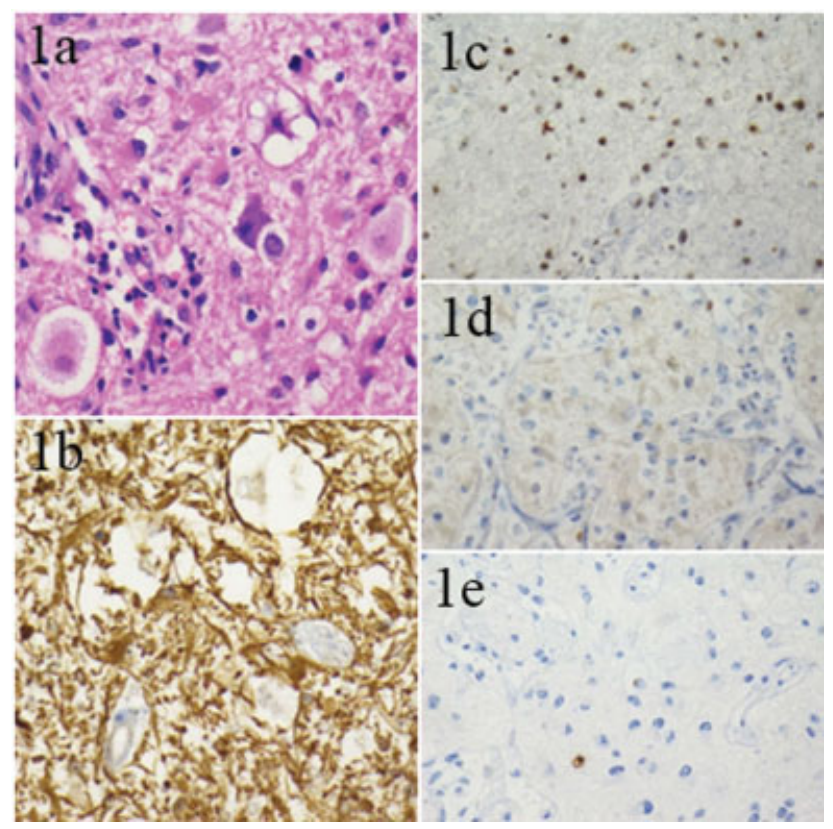

Fig. 1 Pleomorphic xanthoastrocytoma (PXA) with spindle to lipidized and highly pleomorphic cells, eosinophilic granular bodies, and perivascular lymphocytic infiltrate (a); strongly positive glial fibrillary acidic protein (GFAP) (b); retained nuclear expression of the alphathalassemia $x$-linked intellectual disability syndrome ATRX protein (c); no expression of isocitrate dehydrogenase 1 (IDH1) (D); the MIB E3 ubiquitin protein ligase 1 (MIB-1) labeling index is < 1\% (E); a: hematoxylin and eosin (H\&E); b: GFAP; c: ATRX; d: IDH1; e: Ki67. Original magnification (a-e): $40 \mathrm{X}$.

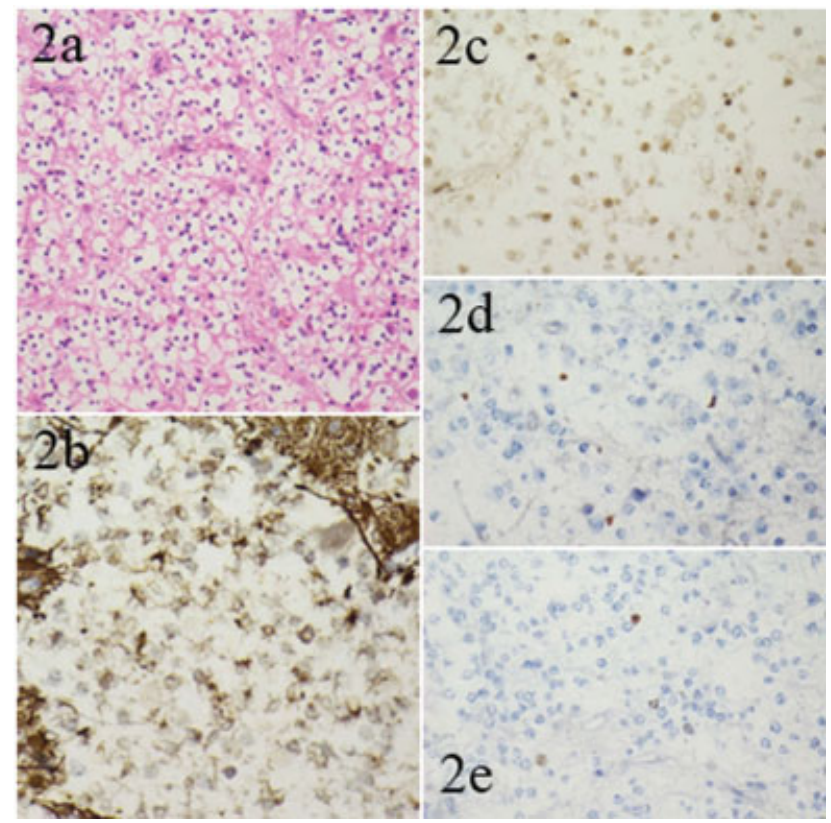

Fig. 2 Oligodendroglial areas with round monomorphic cells with clear cytoplasm arranged in a honeycomb pattern (a); GFAP negative (b); retained nuclear expression of ATRX (c); no expression of IDH1 (d); the MIB-1 labeling index is < $1 \%$ (e); a: H\&E; b: GFAP; c: ATRX; d: IDH1; e: Ki67. Original magnification (b-e): $40 \mathrm{X}$; a: 20X.

cytoplasm arranged in a honeycomb pattern (-Fig. 2a). A delicate chicken-wire network of branching capillaries was noted. No mitosis, necrosis, or endothelial proliferation were observed. The glial fibrillary acidic protein (GFAP) (GA-5) was positive in the PXA component, but negative in the oligodendroglial component (- Fig. 1b\&2b). The alpha-thalassemia $\mathrm{x}$-linked intellectual disability syndrome ATRX (BSB-108) pro-

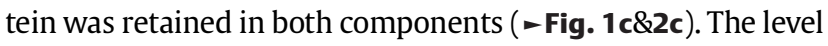
of p53 (DO7) was of $2 \%$ in both the components, and isocitrate dehydrogenase 1 (IDH-1) (H09) was negative in both components ( - Fig. 1d\&2d). The level of KI-67 (SP6) was of $1 \%$ in both the components ( - Fig. 1e\&2e), and synaptophysin (Snp88) and CD34 (QBEnd/10) were positive in the PXA component. Codeletion of $1 \mathrm{p} 19 \mathrm{q}$ (by dual-color fluorescence in situ hybridization [FISH] using Vysis LSI 1p36/1q25 and 19q13/19p13) was performed, and it was negative in both components. The features were compatible with mixed glioma (PXA with oligodendroglioma- like areas, World Health Organization [WHO] grade II). As residual disease was observed on the postoperative scan, the patient was advised to undergo radiotherapy (RT).

\section{Discussion}

The PXA is an astrocytic tumor thought to originate from subpial astrocytes or their precursors. First described in 1973, it was only formally incorporated in the WHO classification in 1993, and it frequently occurs within the first 3 decades of life with the symptom of seizures. Most of these tumors are located in the superficial cerebral cortex, commonly in the temporal and parietal lobes, and present as a complex cystic lesion with associated mural nodules. Isocitrate dehydrogenase mutation 
is usually absent, and ATRX expression is retained. Over $2 / 3$ of these have B-Raf proto-oncogene, serine/threonine kinase (BRAF) mutations. ${ }^{8}$ The rate of mutations in p53 is of up to 25\%. Complete surgical resection and radiotherapy (RT) are the components of the standard care in cases of PXA. This treatment has a good prognosis, with a recurrence-free survival rate of $70.9 \%$ at 5 years. Oligodendroglioma originates from oligodendrocytes or from glial precursor cells. However, the dominance of the oncogenic signaling over the cell of origin determines the of the gliomaphenotype. ${ }^{1}$ Oligodendrogliomas most commonly occur in the frontal lobe, with a peak incidence in fifth and sixth decades of life. These are diffusely infiltrating gliomas with IDH1 or IDH2 mutation, 1p19q co-deletion, and telomerase reverse transcriptase (TERT) promoter mutation. Mutations in the ATRX or tumor protein p53 (TP53) are not observed. The GFAP labels minigemistocytes and gliofibrillary oligodendrocytes, whereas the classic oligodendroglioma cells exhibit minimal or negative GFAP expression. The 5-year survival rate approaches 79.5\%. Although less aggressive, oligodendrogliomas remain incurable, despite the treatment with RT and chemotherapy.

The histopathological diagnosis of mixed/composite glioma is based on the presence of two morphologically distinct glial tumors either diffusely mixed or separated, although the latter is rare. ${ }^{5}$ If these components are genetically distinct, they are termed collision tumors. The 4 commonly used molecular parameters to categorize diffuse gliomas are absence/presence of IDH mutations, 1p/19q co-deletion, TP53 mutation and ATRX loss. Sahm et al. ${ }^{9}$ re-evaluated 43 cases of OA using these 4 paramaters, and reclassified 31 as typical oligodendrogliomas and 11 as DA. Only 1 case had partial 1p19q loss, with IDH1 mutation, ATRX loss, and p53 expression. $^{9}$

Four cases of composite PXA/oligodendroglioma have been reported in the literature, and are summarized in - Table 1. The ages of the partients ranged from 18 to 48 years. Two of the tumors were located in the temporal lobe, and the other two, in the parieto-occipital and frontal lobes. The case herein presented is similar to the case described by Perry et al. ${ }^{4}$ because both were $1 \mathrm{p} 19 \mathrm{q}$ codeletion negative favoring that oligodendroglial component may be arising from PXA; however in the case reported by Perry et al., ${ }^{4}$ PXA the formed a minor component, and in the case reported here it was a predominant component. Moreover, the negative expression of IDH1 in the oligodendroglial component suggests that it may arising from the PXA. The case described by Murakami et al. ${ }^{6}$ had oligodendroglioma as the predominant component with its classical molecular signature, suggesting that the PXA-like microlesion arose from the oligodendroglioma.

Yamada et al. ${ }^{7}$ described a case of combined PXA/infiltrating astrocytoma in which both components showed IDH R132H mutation, but BRAF V600E mutation was only observed in the PXA component and concluded it as intratumoral heterogeneity and clonal evolution. Dualgenotype OAs are also assumed to arise from an IDH-mutant cell of origin, which later, during tumorigenesis develops morphologically distinct subpopulations of tumor cells with astrocytic and oligodendroglial genotypes. ${ }^{1}$ Aisner et al. ${ }^{8}$

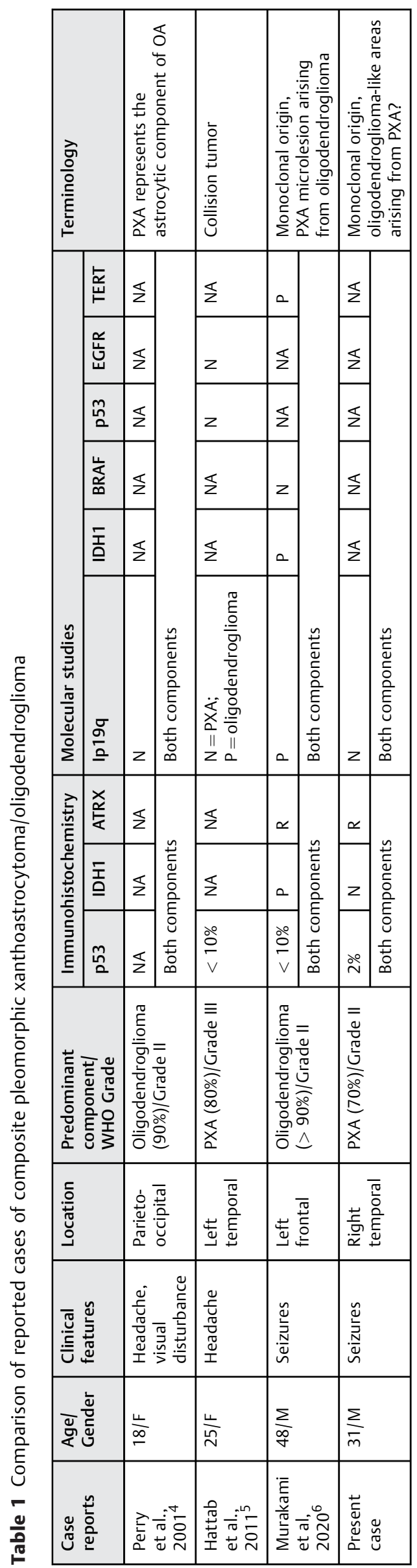


described three cases of composite pleomorphic xanthoastrocytoma-epithelioid glioneuronal tumor (PXA-EGT) and found BRAF mutation in both components, which suggests a common origin.

To conclude, the histogenesis of combined PXA and oligodendroglioma is unknown. All composite gliomas should be subjected to molecular studies in order to enhance our understanding about their tumorigenesis. The case herein reported suggests that PXA can change its morphology to oligodendroglioma. An integrated approach can provide further insights into gliomagenesis.

\section{Contribution of the Authors}

1 Deepa Goel - data analysis, manuscript preparation, manuscript editing.

2 - literature search, manuscript review.

3 - manuscript review.

Guarantor - Dr. Deepa Goel.

Conflict of Interests

The authors have no conflict of interest to declare.

\section{References}

1 Louis DN, Ohgaki H, Weistler OD, Cavenee WK, Eds. WHO classification of tumours of the central nervous system (Revised 4th edition). IARCLyon 2016

2 Perry A, Giannini C, Scheithauer BW, et al. Composite pleomorphic xanthoastrocytoma and ganglioglioma: report of four cases and review of the literature. Am J Surg Pathol 1997;21(07): 763-771

3 Ishizawa K, Terao S, Kobayashi K, Yoshida K, Hirose T. A neuroepithelial tumor showing combined histological features of dysembryoplastic neuroepithelial tumor and pleomorphic xanthoastrocytoma-a case report and review of the literature. Clin Neuropathol 2007;26(04):169-175

4 Perry A, Scheithauer BW, Szczesniak DM, Atkinson JL, Wald JT, Hammak JE. Combined oligodendroglioma/pleomorphic xanthoastrocytoma: a probable collision tumor: case report. Neurosurgery $2001 ; 48(06): 1358-1361$

5 Hattab EM, Martin SE, Shapiro SA, Cheng L. Pleomorphic xanthoastrocytoma and oligodendroglioma: collision of 2 morphologically and genetically distinct anaplastic components. J Neurosurg 2011;114(06):1648-1653

6 Murakami C, Ikota H, Nobusawa S, et al. Oligodendroglioma showing pleomorphic xanthoastrocytoma-like perivascular microlesion: With IDH1, TERT promoter mutation and $1 \mathrm{p} / 19 \mathrm{q}$ codeletion detected in both components. Pathol Int 2020;70(01): 40-46

7 Yamada S, Kipp BR, Voss JS, Giannini C, Raghunathan A. Combined "infiltrating astrocytoma/pleomorphic xanthoastrocytoma" harboring IDH1 R132H and BRAF V600E mutations. Am J Surg Pathol 2016;40(02):279-284

8 Aisner DL, Newell KL, Pollack AG, et al. Composite pleomorphic xanthoastrocytoma-epithelioid glioneuronal tumor with BRAF V600E mutation - report of three cases. Clin Neuropathol 2014;33 (02):112-121

9 Sahm F, Reuss D, Koelsche C, et al. Farewell to oligoastrocytoma: in situ molecular genetics favor classification as either oligodendroglioma or astrocytoma. Acta Neuropathol 2014;128(04): 551-559 